(a) Nausea and Vomiting.-Menopausal women are specially sensitive to oestrogens and therefore particularly liable to exhibit "toxic" effects. For instance, $1 \mathrm{mg}$. of stilboestrol (or its equivalent) daily will induce nausea in most menopausal women. If the patient shows intolerance, even to minimal effective doses, some other oestrogen should be employed, preferably a natural oestrogen such as "strogene."

(b) Uterine Bleeding.-Although this is almost certain to take place if large doses are employed, it not infrequently occurs if small doses (even as low as $0.1 \mathrm{mg}$. of stilboestrol daily) are used continuously for a prolonged period. If this happens, treatment should be discontinued until the bleeding ceases, and then readministered in courses of three weeks with a week's interval between successive courses, during which a withdrawal bleeding may appear.

(c) Other Side-effects.-Water and salt retention is a feature of intensive oestrogen therapy. It gives rise to increase in weight, often accompanied by a bloated feeling. Pain, tenderness, and swelling of the breasts may also develop during continued oestrogen treatment, especially if unnecessarily large doses are used.

There is no convincing evidence that exogenously administered oestrogens are carcinogenic. Nevertheless, it is obviously undesirable to submit a menopausal woman, who is specially sensitive, to excessive doses of oestrogens for prolonged periods.

\section{Artificial Menopause}

Experience has shown that any interference with, or resection of, ovarian tissue increases the likelihood of precipitating the climacteric syndrome, whereas removal of all ovarian tissues renders this practically certain. Furthermore, the severity of the symptoms of the artificial menopause at whatever age it is performed is likely to be considerably greater than that of the natural menopause. Not only are the hot flushes apt to be very frequent but also so severe that the patient is repeatedly drenched in sweat and quite unable to obtain any continued sleep at nights. In addition to this it is not unusual for the symptoms of depression to be especially marked, leading in many cases to severe depressive psychoses and in some instances to involutional melancholia. Every effort should therefore be made by gynaecologists to conserve all possible ovarian tissue, and, if it is decided to abandon the conservative treatment of metropathia, hysterectomy may be preferable to a radium menopause.

\section{THE CHEMOTHERAPY OF CANCER EXPERIMENTS WITH NEW COMPOUNDS}

A lecture on "The Chemotherapy of Cancer" was delivered to the Fine Chemicals Group of the Society of Chemical Industry at the Royal Institution on November 13 by Professor Alexander Haddow, director of the Chester Beatty Institute, the Royal Cancer Hospital.

The great majority of cases of cancer at present, said Professor Haddow, were treated either by surgery in an attempt to extirpate and remove the tumour cells, or by radiotherapy to destroy the cells in situ. Each of these methods had its limitations, and in any case it would clearly be advantageous, particularly as widespread dissemination was an outstanding feature of the disease, if a less local and more general control of malignancy, such as could presumably be brought about by chemical means, were possible.

This had been well recognized from the earliest beginnings of the study of cancer. Ventures in chemotherapy, of a kind, long antedated the modern developments of surgery. Thus at different times in the past there had been applied such agents as belladonna, aconite, mercury, antimony, and arsenic. Many of these chemical applications had a merely caustic action, as, for instance, in the local use of concentrated acids and alkalis and various metals and metalloids. Arsenic had an action which, though not specific, was less indifferent than the others, and the effects of arsenic in the treatment of cancer had been studied more continuously than those of any other agent.

The chemotherapy of cancer was thus of ancient date; interest in it was by no means a recent development. Nevertheless, there had been during the last few years a new burst of activity, arising very largely from a similar increase of interest in more fundamental work in the cancer field. as a whole. In fact, more had been accomplished during the last 10 years than in any other period, though the greater part still remained to be achieved.

\section{The Cancer Cell}

Cancer, Professor Haddow continued, was perhaps the most difficult of all biological problems. The cancer cell was simply a modification of the normal cell. Conversion to malignancy of the normal cell might possibly be brought about by a subtle and elusive change of enzyme constitution quite unaccompanied by any grosser alterations affecting protein structure. On this account there was little or no protective reaction on the part of the body or host, such as occurred in parasitic infections. The malignant cell also appeared to be highly stable in its properties, as was shown by the manner in which its newly acquired characters were transmitted and maintained quite indefinitely. In many ways the processes of cell division were reminiscent of a self-propagating mechanism varying in the degree of control to which it was subject.

It had been suggested that the new configuration of the properties which distinguished the cancer cell from its predecessor might be expressed in terms of energy level or level of organization. The transition from normal to malignant appeared to be by way of intermediate cell types. Undoubtedly the normal cell was highly controlled in contrast to the cancer cell, in which the process of cell division might be regarded as almost autonomous. Nutritionally the normal cell might be regarded as highly dependent, and the malignant cell much less so, if not possibly in many ways autosynthetic.

The cancer cell was a unique development of a specific cell type in an adaptive response to unfavourable conditions, and this was only one of the inherent difficulties which confronted any attempt at chemotherapy. Chemotherapy was expected to undo what could almost be regarded as a natural process.

\section{Sex Hormones}

In face of these difficulties it was perhaps not surprising that such developments in this field as had taken place in recent years had been accomplished without much thought of practical application, mostly as by-products of more important work. This was certainly so in the endocrine control of cancer of the prostate, which was of great importance as the first indication that the autonomy of the cancer cell was not necessarily complete and that it was in fact open to attack by chemotherapy. This development was very largely due to Huggins and his coworkers at the University of Chicago, and followed preliminary studies on normal and hyperplastic prostate glands of dogs. It was very greatly influenced, as Huggins had told him many times, by Huggins's studies at the Lister Institute in London on phosphatase.

In the special case of cancer of the prostate pronounced therapeutic effects were produced by the administration of a natural or synthetic oestrogen, stilboestrol being the synthetic substance most frequently used. In favourable circumstances improvement consisted of a decrease in size of the primary tumour, possibly regression of metastases in bone, improvement in the blood picture, gain in weight, and relief of pain. All these changes were accompanied by an increase in the sense of well-being, and not a few patients, from being confined to bed, became ambulatory and resumed an active life.

This therapy had now been employed for a sufficiently long time to make it clear that, while cancer of the prostate certainly could not be cured by this means, there was equally no doubt that the foci of the disease became quiescent and life was 
prolonged. Again, in a small proportion of cancers of the breast treated by oestrogen there was a most dramatic temporary response. In this connexion it had been interesting to learn of recent investigations in which a favourable response of cancer of the breast or of the cervix had been materially increased by an almost continuous administration of large amounts of watersoluble oestrogen intravenously. There was no doubt that if the mechanism could be elucidated it would be of great importance. A further example of chemotherapy acting through alteration of the hormonal environment of the tumour was the administration of the male sex hormone in cancer of the female breast. There was a curious relationship here-namely, that amelioration was more readily brought about by testosterone in cancer of the breast in women before the menopause, while the beneficial effects from oestrogen were relatively more frequent in post-menopausal cases.

\section{Urethane}

The lecturer next described investigations with urethane. Among other conditions in which the action of urethane had been tested and unusually gratifying responses had been forthcoming was myelomatosis. The action of urethane was characterized by an extremely high degree of chemical specificity, and investigators had been very much concerned to decipher the biochemical mechanism of its action. One of the various alternatives which might be considered was that the substance might renovate some deficiency in the chemical differentiation of the cell. The already extensive literature on the action of urethane had amply confirmed the original findings, but so far had added little to the vital problem of mechanism. There was no question that when that problem was solved it would again have very great significance for the understanding of malignant growth as a whole.

These investigations had one curious feature, namely, that while the effects of urethane were first of all observed in the plant cell and later in animal material, and while it was the latter findings which justified and led to the study of the action of urethane in cancer in man, the characteristic effects in leukaemia were detected solely as a result of clinical observation. The various leukaemias in the mouse and rat were relatively refractory to the influence of urethane, and the remarkable effect in the human might have eluded discovery if attention had been directed to the animal alone. That illustrated the hazards of such work.

The lecturer next mentioned folic acid. Here again the clinical effects were not sufficiently lasting, yet the observation of its action was of fundamental interest and one which would certainly play some part in extending the knowledge of the nutritional basis of cell division.

\section{Nitrogen Mustards}

Professor Haddow came next to a different group of substances - the so-called nitrogen mustards, the biological effects of which on tissues in an active state of proliferation were recognized. During the war they had been tried in Hodgkin's disease and various malignant conditions, in which they had undoubtedly a limited therapeutic action, and it seemed not unreasonable to expect to improve the therapeutic effect by chemical modifications. During the last four years some 250 variants had been tested at the Royal Cancer Hospital, and a number of compounds had been prepared, some of which had perceptible advantages in reduced toxicity. He mentioned in particular the chlorethyl derivative of $\beta$-naphthylamine. Recent reports indicated that substances of thi - type might represent a slight advance, especially in the treatment of such conditions as chronic lymphatic leukaemia. Part of the advantage related, not to any marked increase in inherent activity, but rather to ease of administration and relative absence of serious toxic effects. These compounds, like $x$ rays, could be responsible for nuclear damage, production of mutations, and other effects hitherto associated with ionizing radiations. The lecturer showed certain of these effects in mice. Quantitative comparison of various biological effects induced chemically and by radiation, both in vivo and in vitro, showed a correspondence greater than would seem likely on the grounds of chance alone. The comparison was reinforced by the more recent finding that mustards of this type, again like $x$ and gamma radiation, could also under suitable experimental conditions induce both cancer and mutation. This must be regarded as an important contraindication to the use of them in younger subjects still in the reproductive phase.

Finally the lecturer touched on the recent developments concerning the effects of A.C.T.H. and cortisone in lymphosarcoma, chronic lymphatic leukaemia, acute leukaemia, and certain neoplastic disorders. Again it would seem that any therapeutic action here was subject to obvious limitations and accompanied by certain disadvantages and side-effects; but these observations marked a stage towards an eventual understanding of the different means, direct or indirect, by which the growth of the cancer cell could be affected.

Cancer was almost certainly an error of differentiation. There was very little doubt that it was in this field of cell differentiation that the eventual solution of the problem would be found. At the same time, useful progress could be achieved short of this ideal. It was true that. what Lord Horder had called the distinction between prolonging life and prolonging the act of dying must be borne in mind, but when the infinite series of modifications which were within the range of chemistry were considered, together with the great resources of endocrinology and immunology as yet quite untapped, the age of miracles did not seem to be past.

\section{THE SIR CHARLES HASTINGS LECTURE SIR LIONEL WHITBY ON PREVENTION OF DISEASE}

The first Sir Charles Hastings Lecture to be given since before the war was delivered in the Great Hall of B.M.A. House on November 22. The lecturer was Sir Lionel Whitby, regius professor of physic at Cambridge, and a former President of the Association, and the chair was taken by Dr. Charles Hill, M.P. Accompanying them on the platform were Lady Whitby, Sir William Douglas, Permanent Secretary to the Ministry of Health, Sir Henry Souttar, and others. The hall was entirely filled.

Sir Lionel Whitby, whose subject was "Can Disease be Prevented ?", said that it was necessary at the outset to appreciate how great was the scope of preventive medicine and how many diseases could be prevented. There was indeed hardly anything in the world which did not affect health directly or indirectly; hence the ponderous size of the Ministry of Health, which had to do with drains, sewers, housing, and the huge field of industrial and occupational diseases. many of them with picturesque names such as miner's phthisis, wool-sorter's disease, chimneysweep's cancer, and the like. All these were preventable. Nor were such diseases confined to the labouring classes. They all knew of clergyman's sore throat, boxer's ear, and-a disease frequent when he was young-student's elbow.

\section{Ancient Medicine}

Medicine had been in existence for some 2,000 years. At different periods its practice had been variously based upon demonology, witchcraft, appeasement of divine wrath, folklore, shrewd observation of effects without any real knowledge of cause, and also upon proved knowledge such as was now claimed. Demonology as a basis of medicine was very ancient. It formed the background of Egyptian medicine. There was a papyrus of 1500 B.C. which contained incantations and spells for driving out the devil. Disease was believed to be due to devil-possession. Was there not some resemblance between that and the modern treatment by suggestion which was given to psychoneurotic patients? Folklore likewise was very old. It included prevention by charms, amulets, and herbs, and was the basis of herbalism as it existed to-day. Some of it was sound, some artistic, some revolting; most of it was sheer charlatanism. But the herbalist for large sections of the public satisfied the instinctive craving for the unusual, the miraculous, the search for elixirs of life and perpetual youth. Many a time when he was in practice in Harley Street he 